Revista Eletrônica

REF - ISSN 1808-0804 Vol. VI (3), 23 - 35, 2009

\title{
VIGABATRINA: MECANISMO DE AÇÃO, EFEITOS FARMACOLÓGICOS, ESTUDOS CLÍNICOS E REAÇÕES ADVERSAS
}

Vigabatrin: mechanisms of action, pharmacological effects, clinical studies and adverse reactions

\section{Elisângela Pascoal da Silva' ${ }^{1}$ e Rivelilson Mendes de Freitas²}

${ }_{1}^{1}$ Discente do Curso de Pós-graduação em Farmacologia Clínica da Faculdade Ateneu, Fortaleza, Ceará;

2Professor Adjunto do Setor de Farmacologia da Universidade Federal do Piauí - UFPI, Rua Cícero Eduardo, s/n, Junco, Picos, 64.600-000, Piauí.

E-mail do autor para correspondência: rivelilson@ufpi.br

Recebido em 15/04/2009 - Aceito em 03/09/2009

RESUMO: A vigabatrina é um análogo estrutural do ácido $\mathrm{Y}$-aminobutírico usado no tratamento da síndrome de West, uma forma de epilepsia grave específica da infância, que se caracterizam pela tríade espasmos, atraso neuropsicomotor e hipsarritmia ao eletroencefalograma. $O$ objetivo do presente trabalho foi conduzir uma revisão de literatura sobre os aspectos farmacológicos da VGB, incluindo o mecanismo de ação que possa explicar os efeitos neurotóxicos e a toxicidade aguda e em longo prazo. Métodos: Para tanto foi realizada uma revisão da literatura usando as palavras-chaves vigabatrina, antiepilépticos, toxicidade ocular e intoxicação, por intermédio do MEDLINE e LILACS. A busca inclui todos os artigos publicados no período entre 1937a 2008. Resultados: Ainda existem muitas questões sem respostas sobre a farmacologia da VGB e a fisiologia dos efeitos tóxicos dessa substância. A simples descrição do mecanismo de ação é insuficiente para explicar todos os efeitos induzidos pela VGB. Conclusão: Existem poucas informações na literatura sobre a farmacologia e o mecanismo de ação da VGB que possam explicar os efeitos neurotóxicos e outros efeitos. São necessários mais estudos para que o profissional de saúde possa obter informações e conhecimento, a fim de combater os efeitos tóxicos da VGB.

Palavras chaves: Vigabatrina, Antiepilépticos, Toxicidade Ocular, Intoxicação.

ABSTRACT: The vigabatrin is a structural analogue of $y$-aminobutiric acid used in the treatment of West syndrome, a specific form of severe epilepsy in childhood, characterized by the triad spasms, 
Freitas, R. M. et al./Revista Eletrônica de Farmácia Vol 6(3), 23-35, 2009.

delayed neuropsychomotor hipsarritmia and the electroencephalogram. The objective of this study was to conduct a literature review on the pharmacological aspects of VGB, including the mechanism of action that may explain the retinotoxic effects and acute toxicity and long term. Methods: For this, a literature review was using the keywords vigabatrin, antiepileptics, and retinotoxicity poisoning through the MEDLINE and LILACS was done. The search includes all articles published in the period 1937 to 2008. Results: There are still many questions without answers on the pharmacology and physiology of VGB toxicity of this substance. A simple description of the mechanism of action is insufficient to explain all the effects induced by vigabatrin. Conclusion: There is little information in literature on the pharmacology and mechanism of action of VGB which may explain the neurotoxic effects and other effects. Further studies are required for the health professional to obtain information and knowledge to combat the toxic effects of VGB.

KEY WORDS: Vigabatrin, Antiepileptics, Retinotoxicity, Poisoning. 
Freitas, R. M. et al./Revista Eletrônica de Farmácia Vol 6(3), 23-35, 2009.

\section{INTRODUÇÃO}

A descoberta no final da década de 50 novas drogas antiepilépticas e sua utilização na prática clínica trouxeram um avanço importante no tratamento e no entendimento de possíveis mecanismos subjacentes à epilepsia. Tornou a epilepsia um problema médico passível de tratamento, semelhante a outras doenças como o diabetes e a hipertensão arterial. A partir da década de 60 começaram a surgir novas drogas antiepilépticas. Embora mais eficazes, apresentavam efeitos colaterais indesejáveis causados pela inespecificidade de sua ação farmacológica e eram potencialmente letais em casos de superdosagem (SCHMIDT, 1998; PUTANM \& MERRIT, 1937, GUERREIRO, 2001). Nas últimas décadas surgiram novas classes desses medicamentos para 0 tratamento sintomático da epilepsia com maior segurança e eficácia. Ainda não está claro se a medicação interfere na história natural da condição de base. O risco de o paciente apresentar crises no período em que faz uso da medicação é menor e, assim, melhora claramente a qualidade de vida dos pacientes com epilepsia.

Até o final da década de 80 existiam no mercado essencialmente seis fármacos antiepilépticos: fenobarbital, primidona (cujo principal metabólito é o fenobarbital), fenitoína, carbamazepina, valproato de sódio e etosuximida. Este último fármaco apenas se utiliza em epilepsias de ausências e foi largamente substituído pelo valproato, passando a ser de venda exclusiva nas farmácias hospitalares: os barbitúricos deixaram de serem fármacos de primeira linha pelos efeitos cognitivos que provocam; a fenitoína é também pouco utilizada pelos efeitos secundários. Utilizam-se ainda benzodiazepinas, embora não de primeira linha e, por regra, em associação. A partir dos anos 90 surge uma série de novos fármacos. Na sua maioria nascem de uma investigação orientada para a epilepsia e começam a ser testados nas epilepsias parciais do adulto em terapêutica de associação (ALVES, 1999).

\section{METODOLOGIA}

Neste contexto fez-se uma revisão bibliográfica sobre a VGB. Descreveram-se a estrutura química, mecanismo de ação, posologia, forma de administração, indicação, estudos clínicos, farmacocinética, reações adversas, interações medicamentosas, retinoxicidade, efeitos agudos e crônicos da intoxicação, contra-indicações e recomendações. O mecanismo de ação foi relatado a fim de justificar os efeitos farmacológicos e as possibilidades terapêuticas de reverter às principais complicações associadas ao uso de VGB. Realizou-se uma revisão sistemática de 1937 até 2008 por meio do MEDLINE e LILACS, usando as seguintes palavras chaves: vigabatrine, anticonvulsant, pharmacocinetic e intoxication. Foram selecionados artigos publicados entre 1937 a 2008. Todos os artigos encontrados, preferencialmente artigos de revisão, revisões sistemáticas e ensaios clínicos foram utilizados. 


\section{ESTRUTURA QUÍMICA E MECANISMO DE AÇÃO}

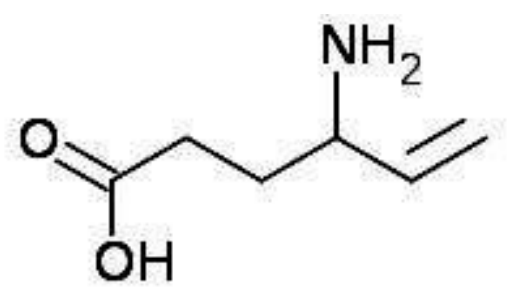

A vigabatrina foi descoberta em junho de 2005 por investigadores da Merrell Recherche, em Estrasburgo, França, após a tentativa de inibir especificamente a enzima GABA transaminase. Assim, desenvolveu-se a VGB ou gama vinil GABA (GUERREIRO, 2001) e lançada no mercado farmacêutico como uma nova droga antiepiléptica. A VGB segundo a IUPAC denomina-se de ácido 4-aminohex-5enóico tem fórmula estrutural análoga ao ácido Y-aminobutírico (GABA), o principal neurotransmissor inibitório do sistema nervoso central. A VGB inibe de forma irreversível a enzima GABA-transaminase (GABA-T), enzima que degrada o GABA promovendo aumento dos níveis deste neurotransmissor. Como a ligação da VGB com a enzima GABA-T é irreversível, sua meia-vida não é importante para a sua eficácia, já que a reciclagem de GABA é muito mais lenta, em torno de três dias. Portanto, a VGB modifica o catabolismo do GABA e exerce sua ação antiepiléptica pelo aumento da concentração do GABA nas sinapses do SNC (ROTTA, et al., 2003).

\section{POSOLOGIA E ADMINISTRAÇÃO}

A literatura registra que a VGB pode ser administrada 1 ou 2 vezes ao dia com água, antes ou depois das refeições. Moraes e colaboradores no ano de 2007 relatam que em adultos, a dose inicial recomendada é de $2 \mathrm{~g}$ que deve ser associada à droga antiepiléptica em uso pelo paciente. Se necessário, a posologia pode ser aumentada ou reduzida gradualmente em frações de 0,5 a $1 \mathrm{~g}$ semanalmente ou a intervalos maiores, dependendo da resposta clínica ou da tolerabilidade. Aumentos na posologia acima de 4 g/dia, em geral, não resultam em maior eficácia. O uso pediátrico em crianças de 3 a 9 anos é indicado na dose inicial $1 \mathrm{~g}$ diariamente. Já em crianças maiores de 10 anos a dose recomendada diariamente é de $2 \mathrm{~g}$ apresentando uma dose média diária de 45 $\mathrm{mg} / \mathrm{kg} / \mathrm{dia}$ de VGB (100-150 mg/dia) associado com ACTH (10 UI/dia). Esses estudos mostram que $42 \%$ dos pacientes responderam à VGB e $74 \%$ ao ACTH; o índice de recidiva durante três meses de tratamento não foi diferente (HENRIQUE-SOUSA, et al., 2007).

Em espasmos infantis associados à esclerose tuberosa, VGB é considerada droga de primeira escolha, entretanto, em uma revisão de trabalhos publicados sobre VGB e ACTH em espasmos infantis por esclerose tuberosa, os resultados foram positivos em $72 \%$ com VGB e em $73 \%$ dos pacientes tratados com ACTH. Outra revisão indicou cessação completa dos espasmos em 95\% dos pacientes (HENRIQUE-SOUSA, et al., 2007). A VGB, também é indicada no combater ao vício induzido pela cocaína (GRAHAM, 1979; BUTLER, 1989). Um estudo clínico de fase 2 realizado 
Freitas, R. M. et al./Revista Eletrônica de Farmácia Vol 6(3), 23-35, 2009.

sobre os efeitos da VGB demonstrou resultados benéficos contra o vício associado à cocaína e a metanfetamina (GRAHAM, 1979; BUTLER, 1989).

\section{INDICAÇÃO}

Diante do exposto, a literatura registra que entre os antiepilépticos, a VGB é indicada principalmente para 0 tratamento dos espasmos infantis na Síndrome de West (SW). Pode, ainda, ser usada em epilepsias generalizadas graves, incluindo a síndrome de Lennox-Gastaut (MORAES, et al., 2005). A VGB também é indicada para o tratamento adjuvante de crises parciais simples e complexas em pacientes refratários, no tratamento da Síndrome de West associado à esclerose tuberosa, idiopática ou sintomática após o uso do hormônio adrenocorticotrófico (ACTH), quando os espasmos infantis persistirem ou quando houver recidiva (REYNOLDS, 1990).

\section{ESTUDOS CLÍNICOS}

Estudos farmacológicos sobre a indicação dessa droga mostram que nos espasmos infantis, a VGB é considerada uma droga de boa eficácia. Outros estudos em crianças com espasmos infantis sem resposta a corticosteróide ou outras drogas antiepilépticas mostraram que $43 \%$ delas apresentam completo controle das crises com VGB. A eficácia parece ser ainda maior nos espasmos secundários à esclerose tuberosa. Doze dentre 14 crianças com espasmos infantis e esclerose tuberosa que utilizaram VGB mostraram controle completo dos mesmos. Vários estudos concluíram que a VGB deve ser considerada como a droga de primeira escolha no tratamento da SW não só pela eficácia igual ou maior que $\mathrm{ACTH}$, como também pelo menor índice de efeitos colaterais graves (REYNOLDS, 1990). A VGB é eficaz para tratamento da Síndrome de West (espasmos infantis), tanto idiopática como criptogênica, associado à esclerose tuberosa (HENRIQUE-SOUSA, et al., 2007).

A VGB foi utilizada especificamente para SW como terapia adjuvante a drogas antiepilépticas (CHIRON, et al., 1991). Neste estudo, 29 crianças (43\%) evoluíram com completa remissão dos espasmos e desaparecimento da hipsarritmia no eletro encefalograma. A VGB foi utilizada em monoterapia em quinze lactentes com SW, obtendo cessação dos espasmos em quatro pacientes $(26,6 \%)$ e melhora das crises em sete pacientes $(46,6 \%)$ nos primeiros 4 a 6 meses (ANTONIUK, et al., 1999). Um estudo multicêntrico na Europa com 192 lactentes demonstrou a supressão dos espasmos em $68 \%$ dos lactentes. Ao final do estudo 96 lactentes (50\%) permaneciam livres dos espasmos com o uso da VGB (HARDING, et al., 1997). Outro estudo duplo-cego, placebocontrolado, realizado com 40 crianças portadoras de SW demonstrou a eficácia clínica da VGB.

Após 24 semanas do estudo, dos 36 pacientes 15 (42\%) ficaram livres dos espasmos durante a VGB com monoterapia. Outro estudo mostrou sua eficácia para crises parciais simples e complexas em adultos e crianças, com ou sem generalização secundária. Entretanto, seu efeito é maior nas crises parciais do que na generalização secundária. Sua eficácia para estes tipos de epilepsia foi demonstra tanto como droga adicional como 
Freitas, R. M. et al./Revista Eletrônica de Farmácia Vol 6(3), 23-35, 2009.

em monoterapia em adultos e crianças. $\mathrm{Na}$ maior parte dos ensaios, vigabatrina foi comparada a placebo ou à carbamazepina. Em alguns ensaios, a vigabatrina foi mais bem tolerada em virtude da sedação causada pela carbamazepina. O controle das crises por ambas as drogas não apresentou diferenças significativas na maior parte dos trabalhos. Em um ensaio clínico, o numero de pacientes livres de crise foi de $58 \%$ para carbamazepina contra $38 \%$ para vigabatrina (GUERREIRO, et al., 2000).

Segundo, Chadwick e colaboradores (1999) as evidências de eficácia favorecem a carbamazepina, não podendo a VGB, portanto, ser indicada como droga de primeira linha em monoterapia. Estudos com monoterapia em crianças também demonstram que a eficácia da VGB e carbamazepina é similar ou favorece esta última. Em um estudo randomizado duplo cego, VGB foi comparada à gabapentina como droga adicional para controle de epilepsia parcial refratária: a diferença de pacientes livres de crises foi inexpressiva e três pacientes tratados com VGB tiveram perturbações dos campos visuais (ALVES, et al., 1999).

Nesta situação, chegou a ser considerada como primeira escolha, entretanto, devido a estudos posteriores ter demonstrado uma relação com retração dos campos visuais passou a ser uma opção terapêutica, e não mais primeira linha. Foi indicada, também, como droga adicional de primeira escolha após recidiva em pacientes tratados com ACTH. Em espasmos infantis idiopáticos (desenvolvimento normal durante a evolução) ou sintomáticos (causa definida) os resultados da VGB são inferiores aos do ACTH ou semelhantes a este (apenas $38 \%$ cessaram os espasmos). Em um estudo, o número de recidivas durante três meses foi alto no grupo tratado com ACTH e ausente no tratado com VGB. No único estudo prospectivo, randomizado, comparando em terapêutica de associação (ALVES, et al., 1999).

Os novos fármacos demonstraram eficácia semelhante à dos antiepilépticos clássicos; a principal diferença surge em termos de efeitos secundários. Na sua grande maioria não são indutores enzimáticos e são mais simples de utilizar por não interferirem com os outros antiepilépticos ou com outra medicação. Apesar dos avanços na pesquisa não dispomos de uma explicação completa e adequada do funcionamento dos antiepilépticos, e assim servimo-nos de hipóteses para entender seu mecanismo de ação. Entre os novos antiepilépticos pode-se citar a VGB que aumenta agudamente a disponibilidade sináptica do neurotransmissor inibitório, o ácido $Y$-amino butírico, através da ação em enzimas específicas. Apesar de essencial, este efeito não explica a demora para se obter resposta clínica, sugerindo que a resolução dos sintomas da epilepsia requeira mudanças adaptativas na neurotransmissão. A principal teoria aceita para explicar tal demora é a da subsensibilização dos receptores pós-sinápticos. O aumento dos níveis de neurotransmissores por inibição da GABA-transaminase ou bloqueio das bombas de recaptura de monoaminas resultar nesta dessensibilização, cuja resolução pode ser correlacionada com o início da melhora clínica. Diante desses dados, o presente trabalho será observado aspectos farmacológicos da VGB, mecanismo de ação proposto, farmacocinética, perfil de efeitos colaterais, interações farmacológicas, contra-indicações e recomendações. 
Freitas, R. M. et al./Revista Eletrônica de Farmácia Vol 6(3), 23-35, 2009.

\section{FARMACOCINÉTICA}

A VGB é administrada por via oral. Apresenta durante a sua distribuição uma alta taxa de ligação às proteínas plasmáticas. Sua absorção no trato digestório apresenta uma concentração máxima entre 3 e 12 horas. É rapidamente absorvida após administração por via oral, é muito solúvel em água. É metabolizada por $\rho$-hidroxilação e conjugação com ácido glicurônico. Apresenta uma meiavida plasmática entre 5 e 9 horas. A VGB é completamente eliminada pelos rins. A droga inalterada é excretada na urina, em $80 \%$, e $20 \%$ na forma de metabólitos inativos (GUERREIRO, 2000). Estudos realizados com a VGB demonstram que após a administração de 1 a $1,5 \mathrm{~g}$ de VGB a concentração máxima é atingida em aproximadamente entre uma e três horas. E sua meia-vida é de aproximadamente 7 horas. Não sofre biotransformação hepática significativa e aparece quase que completamente inalterada na urina (MORAES, et al., 2005).

Segundo a literatura as novas drogas antiepiléticas disponíveis no Brasil inclusive VGB, apresenta perfil farmacocinético adequado, com pequena ligação as proteínas plasmáticas, ausência de indução ou inibição enzimática, e até mesmo excreção renal sem metabolização. Apesar dessas vantagens, a eficácia e os efeitos colaterais destas novas drogas são ainda pouco conhecidos, particularmente em crianças, e algumas delas estão sendo submetidas aos primeiros estudos em monoterapia na população infantil (MORAES, et al., 2005).

\section{REAÇÕES ADVERSAS}

As reações adversas mais freqüentes estão relacionadas principalmente ao Sistema Nervoso Central, provavelmente como conseqüência secundária ao aumento de GABA produzido pela VGB, sendo reversíveis após a suspensão da medicação (MORAES, et al., 2005; REYNOLDS, 1990). Outros efeitos adversos da VGB são geralmente toleráveis e os mais freqüentemente observados em adultos é sonolência, irritabilidade, tontura, fadiga, tremores, parestesias, depressão, ataxia, agitação psicomotora, amnésia, ganho de peso, confusão mental, diarréia, psicose e alterações visuais (MORAES, et al., 2005; REYNOLDS, 1990).

Outros efeitos colaterais também observados são distúrbios hematológicos com diminuição das hemácias; gastrintestinais: constipação, secura na boca, náuseas, vômitos, dor de estômago; sistema nervoso central: tontura, dor de cabeça, depressão, confusão mental, nervosismo, agressividade, distúrbios de memória, dificuldade de concentração, sonolência, cansaço e ainda aumento de peso, displasia gengival, visão dupla e reações alérgicas de pele: urticária, prurido, rash (REYNOLDS, 1990).

Em crianças, os principais efeitos adversos encontrados na literatura foram relatados são excitação e agitação. Pode ocorrer também uma reação paradoxal, com aumento na freqüência das crises mioclônicas (MORAES, et al. 2005). Em nível experimental, em roedores, foi demonstrado que a VGB causa edema intramielínico. E em seres humanos, recentemente, verificou-se que promove constrição do campo visual em 29 a 50\% dos pacientes. A perda da função visual é assintomática e irreversível ou 
Freitas, R. M. et al./Revista Eletrônica de Farmácia Vol 6(3), 23-35, 2009.

incompletamente reversível. O acometimento do campo visual é periférico e predomina no campo nasal. Além da redução do campo visual, a VGB pode ainda comprometer a acuidade visual, a visão colorida e a amplitude dos potenciais obtidos no eletroretinograma (ROTTA, et al., 2003).

Estudos demostram que a interrupção do tratamento com VGB não modifica o comprometimento do campo visual, mas o déficit na acuidade visual, na visão colorida e na amplitude dos potenciais obtidos no eletroretinograma que pode ser revertido naqueles pacientes que apresentavam um comprometimento mínimo de campo visual (MORAES, et al., 2005). Existem trabalhos que demonstram a avaliação do potencial evocado visual em crianças pode substituir a campimetria e demonstrar o acometimento das vias visuais desde a fase inicial. As principais alterações oculares são hipopigmentação da retina, aumento da parede vascular, atrofia óptico e alteração no potencial evocado visual em crianças tratadas com VGB (KOUL, et al., 2001).

Estudos em laboratórios demonstram que a vigabatrina pode induzir uma diminuição no peso de ratos (CHIRON, et al., 1991). Os resultados da pesquisa revelaram que ratos geneticamente modificados quanto à obesidade perderam até $19 \%$ de seu peso total, enquanto que, os animais normais emagreceram de $12 \%$ a $20 \%$, após um tratamento de curta duração com VGB (CHIRON, et al., 1991). Esses resultados sugerem que a VGB pode também apresentar como reação adversa um efeito anorexígeno (MORAES, et al., 2005). O Quadro 1 apresenta as principais reações adversas leves e graves durante o tratamento com a vigabatrina.

Quadro 1: Classificação das principais reações adversas durante o tratamento com a vigabatrina.

\begin{tabular}{|c|c|c|}
\hline $\begin{array}{l}\text { Reações } \\
\text { adversas }\end{array}$ & Crianças & Adultos \\
\hline Leves & $\begin{array}{l}\text { Excitação, agitação, reação } \\
\text { paradoxal, aumento de peso, } \\
\text { efeitos gastrintestinais leves, } \\
\text { constipação, secura na boca, } \\
\text { náuseas, vômitos, dor de } \\
\text { estômago, tontura, dor de cabeça, } \\
\text { depressão, sonolência, cansaço, } \\
\text { reações alérgicas de pele. }\end{array}$ & $\begin{array}{l}\text { Sedação, fadiga, cefaléia, } \\
\text { tontura, confusão, insônia, } \\
\text { irritabilidade, agressividade, } \\
\text { distúrbios de memória, aumento } \\
\text { de peso e psicose. }\end{array}$ \\
\hline Graves & $\begin{array}{l}\text { Constrição persistente do campo } \\
\text { visual, Hepatotoxicidade grave ou } \\
\text { fatal, crescimento gengival. }\end{array}$ & Não há registros na literatura \\
\hline
\end{tabular}




\section{EFEITOS AVERSOS AGUDOS E CRÔNICOS}

Os principais efeitos adversos agudos e crônicos associados ao tratamento com VGB são encefalopatia aguda, anomalias eletroencefalograma, hidrocefalia, deficiência orgânica metabólica, e a insuficiência renal, alterações na arquitetura do sono (CHIRON, et al., 1991).

\section{INTERAÇÕES MEDICAMENTOSAS}

Como descrito anteriormente a VGB não é metabolizada no fígado, não se liga às proteínas plasmáticas, nem tampouco é indutora do sistema enzimático do citocromo P450, portanto, interações com outras drogas são pouco prováveis. As concentrações plasmáticas de carbamazepina, fenobarbital e valproato de sódio, monitorizadas durante estudos clínicos controlados, não sofreram variação significativa com a administração concomitante de VGB (FONSECA, et al., 2000). Entretanto, durante os estudos clínicos controlados, observou-se uma redução gradual de cerca de $20 \%$ nas concentrações plasmáticas de fenitoína. A natureza desta interação ainda não foi completamente elucidada. Poucos estudos avaliaram as interações entre VGB e outras drogas antiepilépticas (FONSECA, et al., 2000). O consumo de álcool, por sua propriedade de indução do metabolismo hepático com redução dos níveis das drogas antiepilépticas e contribuição para diminuição da adesão, e ainda em decorrência da privação do sono pode estar relacionado com aumento no número de crises não apresenta interações com alimentos (KANEKO, 2000).

\section{MECANISMO DE AÇÃO DA TOXICIDADE NO} SISTEMA OCULAR
Toxicidade ocular caracterizada por edema intramielínico e vacuolização celular tenha sido observada em ratos e cães, acreditase que isto não ocorra em primatas (GRAHAM, 1979; BUTLER, 1989). Em termos eletrofisiológicos alguns autores descrevem alterações do eletrorretinograma (nas primeiras seis semanas) sugestivas de disfunção cone e traduzidas por perda dos potenciais oscilatórios. Outros estudos registaram reduções na onda $b$ escotópica sugerindo que a VGB. Pode induzir a uma disfunção retiniana difusa, afetado também o sistema bastonete. No estudo de Harding e colaboradores a diminuição da amplitude do Flicker $30 \mathrm{~Hz}$ estava associada com apresença e grau de gravidade dos defeitos campimétricos atribuídos à terapêutica com VGB (MILLER, et al., 1999).

\section{INTOXICAÇÃO E TRATAMENTO}

Os principais sinais e sintomas da intoxicação a VGB ainda não foram completamente descritos, por se tratar de medicamento novo, conhecem-se muito pouco sobre a superdosagem no homem, tendo sido relatados casos isolados de superdosagem. Com a dose diária de $14 \mathrm{~g}$ durante três dias, após ingestão acidental, observou-se apenas vertigem e tremores transitórios (FONSECA et al., 2000). A literatura demonstra que não há um antídoto específico para o tratamento de intoxicação com VGB. Há relato de uma paciente de 18 anos que ingeriu $30 \mathrm{~g}$ de VGB associado a $250 \mathrm{mg}$ de clorazepato dipotássico em tentativa de suicídio, o que a levou ao estado de coma, persistente por quatro dias com recuperação sem seqüelas. $O$ quadro comatoso foi, entretanto, atribuído mais ao clorazepato do que ao VGB (GUERREIRO, 2005). 
Freitas, R. M. et al./Revista Eletrônica de Farmácia Vol 6(3), 23-35, 2009.

\section{CONTRA-INDICAÇÕES E RECOMENDAÇÕES}

O tratamento com a VGB é contraindicada em pacientes com história prévia de hipersensibilidade. Deve ser usado com cuidado em pacientes com história pregressa de distúrbios comportamentais e psiquiátricos. Não deve ser administrado a mulheres grávidas e durante a lactação. Não existem estudos bem controlados em mulheres grávidas ou durante a lactação. A VGB piora crises generalizadas primárias; provoca aumento de ausências e pode desencadear crises mioclônicas em mulheres grávidas. É contra-indicada em epilepsias mioclônicas e nas crises tônicoclônicas primárias (MORENO, et al., 1999).

A VGB é eliminada pelos rins e, portanto, deve-se ter cuidado quando se administrar à droga a pacientes idosos e, particularmente, a pacientes com clearance de creatinina inferior a $60 \mathrm{ml} / \mathrm{min}$. É recomendável que tais pacientes iniciem o tratamento com VGB com posologias menores e deve ser cuidadosamente observada em relação a efeitos adversos como sedação ou confusão mental. A VGB pode ser administrada antes ou após as refeições (MORENO, et al., 1999).

A perda da função visual é um efeito colateral grave e irreversível, portanto, a VGB deve ter uma indicação restrita em casos comprovadamente eficazes. É aconselhado fazer revisão oftalmológica e campimetria a cada seis meses (MORENO, et al., 1999). O tratamento com VGB não deve retirado abruptamente para não provocar síndrome de abstinência, a posologia deve ser reduzida gradualmente, durante duas a quatro semanas (KRAUSS, et al., 1998). Na maioria dos casos as reações adversas são consideradas leves, no entanto, pode ser necessária a interrupção do tratamento (MORENO, et al., 1999).

\section{CONSIDERAÇÕES FINAIS}

Está claro que o consumo da VGB, segundo estudos clínicos é indicado no controle das convulsões, bem como as alterações eletroencefalográficas em crianças com esclerose tuberosa e síndrome de West. A suspensão abrupta da VGB pode ocasionar crises com síndrome de abstinência. Se for necessária a interrupção do tratamento, é recomendável que se reduza gradualmente a posologia do medicamento por um período de 2 a 4 semanas. Há também relatos de quadro psicótico grave causado pela suspensão abrupta do medicamento. Diante de tais complicações é necessário um acompanhamento médico. Para tanto é fundamental que o profissional de saúde conheça os efeitos psicológicos e toxicológicos provocados pelo uso da VGB. Embora existam dados na literatura sobre os principais efeitos e sintomas das complicações associadas ao uso da VGB, são necessários novos estudos sobre a fisiopatologia e farmacologia da VGB, principalmente para explicar o mecanismo da toxicidade ocular. 
Freitas, R. M. et al./Revista Eletrônica de Farmácia Vol 6(3), 23-35, 2009.

\section{REFERÊNCIAS BIBLIOGRÁFICAS}

ALVES, N.D.; et al. Possible Analgesic Effect of Vigabatrin in Animal Experimental Chronic Neuropathic Pain. Arq Neuropsiquiatr, v. 57, p. 916-920, 1999.

ANDERSON,G.D. Pharmacokinetic, pharmacodynamic, and pharmacogenetic targeted therapy of antiepileptic drugs. Ther Drug Monit; v.30, p.173-180, 2008.

ANTONIUK, A.S.; et al. SÍNDROME DE WEST. Evolução Clínica e Eletrencefalográfica de 70 pacientes e Resposta ao Tratamento com Hormônio Adrenocoticotrófico, Prednisona, Vigabatrina, Nitrazepan e Ácido Valpróico. Arq Neuropsiquiatr; v. 58, p.683-690, 2000.

BUTLER, W.H. The neuropathology of vigabatrin. Epilepsia; v. 30, p. 15-17, 1989.

CHIRON, C.; et al. Therapeutic trial of vigabatrin in refractory infantile spasms. J Child Neurol; v. 6, p. 2552-2558, 1991.

FONSECA, L.M; OLIVEIRA, A.L. Espasmos Infantis: Experiência em Treze Casos. Arq Neuropsiquiatr; v. 58, p. $512-517,2000$.

FREITAS, E.V.; et al. Tratado de Geriatria e Gerontologia, Rio de Janeiro: Editora Guanabara Koogan, 2002.

GRAHAM, D. Neuropathology of vigabatrin. Br J Clin Pharmacol; v. 27, p. 43-53, 1979.

GUBERMAN, A. Monotherapy or polytherapy for epilepsy. Can J.Neurol Sci; v. 25, p. 3-8, 1998.

GUERREIRO, C.A.M, GUERREIRO, M.M. Novas Drogas. In: GUERREIRO, C.A.M, GUERREIRO, CENDES-LOPES, I; São Paulo: Editora Lemos Editorial \& Gráficos Ltda, 2000.

GUERREIRO, C.A.M. Tratamento medicamentoso das epilepsias com novas drogas e complicações de epilepsias crônicas. 2001, p. 823, Dissertação (Livre-Docência), Faculdade de Ciências Médicas da Universidade Estadual de Campinas.

GUERREIRO, M.M. Avaliação da Eficácia e Tolerabilidade da Vigabatrina na Síndrome de West. Arq Neuropsiquiatr; v. 63, p. 469-473, 2005.

GUYTON, A.C, HALL, J.E. Tratado de fisiologia médica. 11 ed., Rio de Janeiro: Editora Elsevier, 2006. 
Freitas, R. M. et al./Revista Eletrônica de Farmácia Vol 6(3), 23-35, 2009.

HARDING, G.F.A. Severe persistent visual field constriction associated with vigabatrin. Br Med J; v. 314, p. 1694-1697, 1997.

HENRIQUES-SOUZA, A.M.M.; LAURENTINO, L.A.J.S.G.; Vigabatrina no Tratamento da Síndrome de West. Arq Neuropsiquiatr; v. 65, p. 144-149, 2007.

KANEKO, S. Epilepsy, pregnancy, and the child. Epilepsia; v. 41, p. 8-13, 2000.

KOUL, R.; et al. Vigabatrin associated retinal dysfunction in children with epilepsy. Arch Dis Child ; v. 85, p. $469-473,2001$.

KRAUSS, G.L.; et al. Vigabatrin-associated retinal cone system dysfunction. Electroretinogram and ophthalmological findings. Neurology; v. 50, p. 614-618, 1998.

LÖSCHER, W; KLOTZ, U. The clinical impact of pharmacogenetics on the treatment of epilepsy. Epilepsia; v.2, p.1-23, 2008.

LUNA-TORTÓS, C.; et al. Several major antiepileptic drugs are substrates for human P-glycoprotein. Neuropharmacology; v.55, p.1364-1375, 2008.

MILLER, N.R.; et al. Visual dysfunction in patients receiving vigabatrin. Clinical and electrophysiological findings. Neurology; v.53, p. 2082-2087, 1999.

MORAES, M.H.P.; et al. Avaliação da Eficácia e Tolerabilidade da Vigabatrina na Síndrome de West. Arq Neuropsiquiatr; v. 63, p. 469-473, 2005.

MORENO, R.A.; et al. Psicofarmacologia de antidepressivos. Rev. Bras. Psiquiatria; v. 21, p. 24-40, 1999.

NEHLIG, A.; et al. Vigabatrin protects against hippocampal damage but is not antiepileptogenic in the lithium-pilocarpine model of temporal epilepsy. Epilepsy Research; v. 47, p. 99-117, 2001.

PUTANAM, T.J.; MERRIT, H.H. Experimental determination of the anticonvulsant properties of some phenyl derivatives. Science; v. 85, p. 525-526, 1937.

RANG, H.P.; et al. Farmacologia. 6a edição, Rio de Janeiro: Editora Elsevier, 2008.

REYNOLDS, E.H. Vigabatrin. British Medical Journal; v. 300, p. 277-278, 1990. 
Freitas, R. M. et al./Revista Eletrônica de Farmácia Vol 6(3), 23-35, 2009.

ROTTA, N.T.; et al. Vigabatrina no Tratamento da Epilepsia de difícil controle em Pacientes com Síndrome de West e Esclerose Tuberosa. Arq Neuropsiquiatr; v. 61, p. 988-990, 2003.

SCHMIDT, D. Drug Trial in Epilepsy. A physician's guide. London: Martin Dunitz; 1998.

SELA, C.E.; TRIMBLE, M.R. Adjunctive therapy in epilepsy with new antiepileptic drugs: is it of any value? Seizure; v. 7, p. 417-418, 1998.

STRIANO, P.; et al. Refractory, life-threatening status epilepticus in a 3-year-old girl. Lancet Neurology; v.7, p.278-284, 2008.

THEODORE, W.H, PORTER, R.J. Removal of sedative-hypnotic antiepileptic drugs from the regimens of patients with intractable epilepsy. Journal of Epilepsy and Clinical Neurophysiology; v.13, p. 320324, 1983.

YACUBIAN, E.M.T., et al. Tratamento da epilepsia na infância. J. Pediatr; v. 78, p. 7557-7559, 2002.

YERBY, M.S. Risks of pregnancy in women with epilepsy. Epilepsia; v. 33, p. 23-27, 1992. 